\title{
Inflammatory markers in temporomandibular joint disorders
}

\author{
JOLANTA KOSTRZEWA-JANICKA', PIOTR JURKOWSKII, MAEGORZATA NĘDZI-GÓRA2, \\ ELŻBIETA MIERZWIŃSKA-NASTALSKA ${ }^{l}$
}

${ }^{1}$ Department of Prosthetic Dentistry, Medical University of Warsaw, Poland

${ }^{2}$ Department of Periodontology, Medical University of Warsaw, Poland

\begin{abstract}
Temporomandibular joint disorders (TMD) have a chronic and progressive character. Multifactorial etiology of the disorders and difficulties in detection of first signs and symptoms caused, in many cases, symptomatic treatment approach. The essential is to establish the sensitive and specific diagnostic methods for early causal treatment possibilities. Clinical examination and imaging techniques do not always allow the full view of the ongoing disease. The concentration of inflammation indicators, such as $C$-reactive protein $(C R P)$ is not a specific tool, which does not indicate any specific disease, but the synovial fluid markers, such as cytokines and proteinases, could allow detect the early bony deterioration in the temporomandibular joint (TMJ), which is not visible on radiographic imaging. Although, the small amount of collected fluid from the joint and specialist laboratory procedures are not regularly obtain in daily practice. Although, early detection of tissues damaged, would enable clinicians to introduce proper treatment and prevent irreversible degenerative changes in TMJ.
\end{abstract}

Key words: temporomandibular joint, internal derangements, osteoarthritis, cytokines.

(Centr Eur J Immunol 2012; 37 (3): 290-293)

\section{Introduction}

The population-based epidemiological studies report that $10-15 \%$ of adults suffering from temporomandibular disorders (TMD) experience pain. In 40-75\% occurs at least one of the symptoms of masticatory system dysfunction [1-3]. Several studies have pointed out that persistent and recurrent pain is a potential etiological factor of psychological discomfort and physical disability, what can lead to limitations in quality of life $[4,5]$. The clinical studies lead to develop of standards and guidelines for diagnosis and treatment, especially the first signs and symptoms of TMD. It is commonly agreed to separate the temporomandibular joint (TMJ) disorders from masticatory muscles disorders, although TMJ and muscles symptoms can occur simultaneously. The common disorders which affect TMJ are disc displacement (with and without reduction) and degenerative joint disease (arthrosis, arthritis) [6]. Both can lead to significant loss of function and joint pain. Pathologic changes of TMJ include inflammation, degeneration and deformation [7]. The natural his- tory of TMD, especially the TMJ diseases, is characterized by tissues adaptation and recoveries from episodes of pain and dysfunction. The most often clinically used diagnostic protocol is the Research Diagnostic Criteria for Temporomandibular Disorders (RDC/TMD) [6]. In the cases of TMJ arthropathy, additionally to clinical examination (RDC/ TMD), the radiographic techniques (panoramic radiography, plain radiography, conventional and computer tomography, magnetic resonance imaging) can help to diagnose the abnormalities of bony or soft tissue $[8,9]$. Although, the diagnosis based on clinical findings is not always confirmed by the findings made through for example magnetic resonance imaging (MRI) [10]. Also, is impossible to detect the first signs of synovium and cartilage damage [11].

Pathophysiology of TMD is still under consideration by many scientific investigators $[12,13]$. The chronic and progressive character of that disorders induce the search for more specific methods for early diagnosis and monitoring of disease progression using the specific and sensitive diagnostic tools.

Correspondence: Jolanta Kostrzewa-Janicka, Department of Prosthetic Dentistry, Medical University of Warsaw, Nowogrodzka 59, paw. XIa, 02-006 Warsaw, Poland, e-mail: Kostrzewa@ stoma-med.net, tel. +48 225021886 


\section{Pathology of temporomandibular joint}

Temporomandibular joint is a synovial joint with articular disc. The structure of TMJ, particularly of the disc, under light microscopy has been described as composed of collagen and elastic fibres with chondroid cells [14-16]. The TMJ structures are mainly covered with fibrous cartilage. The inner surface of joint capsule is the synovial membrane, which produce the synovial fluid supplies the nutritional and metabolic products of the nonvascularized articular tissues and give the joint lubrication [17]. The synovial membrane contains specialized cell types with phagocytic and immunologic capacity. The reduction of the quantity and /or alteration in the quality of the synovial fluid not only impairs the nutritional support of the articular cartilage, but also is associated with loss of cartilage matrix [18].

Temporomandibular joint disc displacement is characterized by an abnormal disc position relative to the condyle and articular eminence, often associated with synovitis [19, 20]. As derangements become more severe, the histologic changes in TMJ are also more intense, ending in osteoarthritis, vs. osteoarthrosis (OA).

Osteoarthrosis is a degenerative disease defined as a focal non-inflammatory disease, characterized by the ongoing process of destruction and repair [18]. An etiological hypothesis of OA is mainly based on the concept of joint overloading, due to, for example parafunctions, loss of posterior teeth, muscles hyperactivity. An abnormal and repetitive mechanical stresses exceed the functional capacity of articular tissues. In TMJ OA the progressive cartilage breakdown is observed, which leads to perforation of the disc and denudation of the subchondral bone. Crepitus is the major clinical sign of this stage. There are the characteristic radiographic signs of TMJ OA, such as osteophytic lipping, cyst formation, flattening of the condyle and eminence, and decreased height of the mandibular ramus. The damaged joints' surfaces may be accompanied by pain, and limited mobility of the joint. Cartilage breakdown is followed by repair process with proliferation of chondrocytes and increased synthesis of matrix collagen and proteoglicans. This explains the time needed for detection of the first signs and symptoms of OA, and lack of signs on radiographic images.

The important participants in the OA are considered cytokines, such as interleukin-1 (IL-1), tumor necrosis factor $\alpha$ (TNF- $\alpha)$ [21]. The cytokines are produced by chondrocytes, synovial macrophages, or synovial fibroblasts [22-26]. The inflammatory process activate enzymes degrading synovium and articular cartilage [27].

It is suggested, that pain intensity is correlated with expression of calcitonin gene-related peptide (CGRP) and substance P (SP) [28, 29]. The concentration of neuropeptides, cytokines, leukotrienes, prostaglandins and catabolic products, identified in TMJ fluid, are correlated with joint pain and presence of surface lesions observed within the joint. Unfortunately, many of these molecules are also involved in remodeling processes affecting TMJ, in normal adaptation [19]. Then, first should be isolating substances involved in adaptation, to be able to identify those engaged in TMJ disease.

In the diagnosis of OA, the radiological evaluation of subchondral bone resorption is usually made. The cartilage erosion in OA or rheumatoid arthritis (RA) is indirectly measured on radiographs by assessment of the joint space width [11]. Osteoarthrosis begins in the cartilage, so on radiographs it is difficult to detect the early stages and evaluate the pro-gress of the intra-articular pathologic status. It takes usually 2-3 years to detect the joint cartilage destruction using radiological methods. Then, sensitive indicators of early metabolic disturbances in cartilage and bone will be very useful.

\section{Inflammatory mediators in the temporomandibular joint synovial fluid}

The TMJ internal derangements and OA are associated with inflammatory process in the synovial membrane and articular cartilage [21]. There are proposed three mechanisms of TMJ degenerative disease: direct mechanical injury, hypoxia-reperfusion injury, and neurogenic inflammation [27]. Mechanical overloading and increased intracapsular hydrostatic pressure decreased fluid transport and blood flow. The physical disruption of molecules and hypoxia can lead to the production of the free radicals. They are extremely reactive and can damage the important molecules in articular tissue and in synovial fluid. Free radicals can increase arachidonic acid metabolism with production of prostaglandins, leukotriens, and matrix degrading enzymes, for example collagenases, stromelysins, gelatinases. In that way the overloading and hypoxia lead to deformation of cartilage matrix and provoke the net tissue loss. On the other hand, the activated peripheral nerves release neuropeptides, such as SP and CGRP, which can evoke an inflammatory response by activation of cytokines (for example: interleukin-1, interleukin-6, tumor necrosis factor- $\alpha$, interleukin- 8 , endothelin-1), and arachidonic acid catabolism. This neurogenic mechanism of inflammation also provides to cartilage and synovium degradation.

It is elucidated, that excess amount of the inflammatory mediators in the synovial fluid of TMJ in patients with TMD is important, and also their characteristics, balance and theirs receptors $[22-26,30]$. There are the mutually protected and regulated mechanisms of inflammation. Cytokines, such as IL- 1 and TNF- $\alpha$, can caused cartilage degradation thorough up-regulation of metalloproteinases (MMP) gene expression, and decreasing chondrocyte compensatory synthesis pathways. Cytokines produced by activated synovicytes, mononuclear cells or by articular cartilage, have their natural antagonists. Soluble forms of tumor necrosis factor receptors (sTNFR) can neutralize the bio- 
logical activities of TNF- $\alpha$, and IL-1 receptor antagonist (IL-ra) is a natural inhibitor of IL-1 activity. There are also naturally occurring anti-inflammatory cytokines such as IL-10, which inhibit the synthesis of IL-1 and TNF- $\alpha$. In TMJ synovium patients with OA there is observed the higher concentration of cytokines and protein compared with control and the imbalance between cytokine and its soluble receptors $[21,22]$. There was found that STNFR were elevated in patients with internal derangements and $\mathrm{OA}$, and that concentrations of soluble receptors of IL-1 and IL-6 were increased in patients with OA compared to the controls [22]. Then, the ratio between cytokines and its soluble receptors can influence the homeostasis and prognosis of pathological process in TMD patients.

Increased levels of cytokines (IL-1, IL-6) and active forms of MMP can suggest the cartilage degradation, which was revealed in patients with diagnosed OA but also in patients with TMJ internal derangements with disc displacement without reduction $[23,31]$. Other study revealed that increased concentration of IL-6 in synovial fluid can reflect degenerative changes of the condyle [25]. The IL-6 can induce the release of proteinases, which destroy cartilage and induce bony resorption. There was detected the correlation among the concentrations of IL-8, IL-6 and TNF- $\alpha$. There was no correlation between the amount of any cytokines and clinical symptoms. Other authors also suggest that IL-1 and stromelysin (MMP3) can be the markers of early bony deterioration, which is not detected by radiograph imaging [24]. It was revealed that in bony degrading stage of internal derangements, the IL- 1 is produced by macrophages from synovium but also by chondrocytes or fibroblasts from articular cartilage, that suggest the possibility of detection the early OA changes.

The inflammatory markers such as TNF- $\alpha$, IL- $1 \alpha$, IL-1 $\alpha$, IL-6, IL-8, various kinds of MMPs should be investigate in aspect of its concentrations but also in aspect of its ratio to their receptors and other mediators [22, 32]. That balance is very important for homeostasis and for treatment prognosis.

\section{Summary}

Multifactorial etiology of TMD forced clinicians to apply wide diagnosis and reversal, conservative treatment [33]. In many cases it is just treatment of symptoms. Then, it is important to research the underlying pathogenesis of TMD to develop tissue-targeted therapies, to stop or even reverse the pathologic processes. However, in causal therapy, precise and early diagnosis is very important. It seems to be very useful to obtain the "markers" of specific TMJ pathology. Compared to radiological methods, the "markers" would provide quicker detection of pathology in its early stages, and would give information about the therapeutic effects, what would be useful in selection of therapy or drugs doses. Taking into consideration the cartilage and bone degradation, the biochemical markers of bone turnover seem to meet the expectations, especially in diagnosis of osteoporosis, bone tumors, osteonecrosis, osteoarthritis, and rheumatoid arthritis. Unfortunately, these markers are sensitive, but not specific. As they visualize all remodeling processes in skeleton, it is very difficult to decide, if the results regard TMJ only. The same problem arises when interpreting concentration of inflammation indicators, such as C-reactive protein (CRP). There are large numbers of different conditions that can increase the CRP level, which does not indicate any specific disease. More information could be obtained from synovial fluid of TMJ, about both cytokines and their receptors, which are involved in the pathogenesis of TMD [22-26]. However, it is very difficult to aspirate the proper amount of synovial fluid from such a small joint, even that recent advances in arthroscopic surgery allowed the direct examination of the TMJ. In both situations, the special equipment and biochemical components are necessary, so it is difficult to apply that procedures in daily practice. Additionally, should be the same assay - systems in laboratories. Although, that studies allowed understand the mechanisms of pathophysiology of the TMJ disorders, and give the assumption for treatment approach. There are also possibilities of applying the electron spin resonance (ESR) in diagnosis of reactive oxygen species (ROS) in the synovial fluid from temporomandibular disorder patients [34]. ROS correspond to free radical species and could exacerbate the inflammatory process and activate the cartilage matrix degrading enzymes, what leads to OA.

According to the present knowledge, the measurement of bone turnover markers together with an assessment of inflammation indicators, and radiological images of TMJ seems to be optimal. This could give the information on the severity of degenerative changes in the joint structures, and the inflammatory process in the course of internal derangements and osteoarthritis.

\section{References}

1. Macfrane TV, Glenny AM, Worthington HV (2001): Systematic review of population - based epidemiological studies of orofacial pain. J Dent 29: 451-467.

2. Ash M, Ramfjord P: Occlusion. $4^{\text {th }}$ ed. WB Saunders Co. Philadelphia, PA, 1995.

3. Carlsson GE, LeResche L (1995): Epidemiology of temporomandibular disorders. In: Sessle BJ, Bryant PS, Dionne R (eds.): Temporomandibular Disorders and Related Pain Conditions. IASP Press, Seattle; 211-226.

4. John MT, Reissmann DR, Schierz O, Wassell RW (2007): Oral health-related quality of life in patients with temporomnadibular disorders. J Orofac Pain 21: 46-54.

5. Türp JC, Motschall E, Schindler HJ, Heydecke G (2007): In patients with temporomandibular disorders, do particular interventions influence oral health - related quality of life? A qualitative systematic review of the literature. Clin Oral Implants Res 18: 127-137. 
6. Dworkin SF, Le Resche L (1992): Research diagnostic criteria for temporomandibular disorders: review, criteria, examinations and specifications, critique. J Craniomandib Disord 6: 301-355.

7. de Bont LG, Dijkgraaf LC, Stegenga B (1997): Epidemiology and natural progression of articular temporomandibular disorders. Oral Surg Oral Med Oral Pathol Oral Radiol Endod 83: 72-76.

8. Usümez S, Oz F, Guray E (2004): Comparison of clinical and magnetic resonance imaging diagnoses in patients with TMD history. J Oral Rehabil 31: 52-56.

9. Westesson PL, Katzberg RW, Tallents RH, et al. (1987): CT and MR of the temporomandibular joint: comparison with autopsy specimens. AJR Am J Roentgenol 148: 1165-1171.

10. Limchaichano N, Nilsson H, Ekberg EC, et al. (2007): Clinical diagnoses and MRI finding in patients with TMD pain. J Oral Rehabil 34: 237-245.

11. Ory PA (2003): Interpreting radiographic data in rheumatoid arthritis. Ann Rheum Dis 9: 597-604.

12. Green CS (2001): The etiology of temporomandibular disorders: implication for treatment. J Orofac Pain 15: 93-105.

13. Cairns BE (2010): Pathophysiology of TMD pain - basic mechanisms and their implications for treatment. Review article. J Oral Rehabil 37: 391-410.

14. Singh M, Detamore MS (2009): Biomechanical properties of the mandibular cartilage and their relevance to the TMJ disc. Review. J Biomechanics 42: 405-417.

15. Thilander B, Carlson GE, Ingervall B (1976): Postnatal development of the human temporomandibular joint. I. A histological study. Acta Odontologica Scan 34: 117-126.

16. Wong GB, Weinberg S, Symington JM (1985): Morphology of the developing articular disc of the human temporomnadibular joint. J Maxillofac Surg 43: 565-569.

17. Mow VC Holmes MH, Lai WM (1984): Fluid transport and mechanical properties of articular cartilage: A review. J Biomech; 17: 377-394.

18. Stegenda B, de Bont LGM, Boering G (1989): Osteoarthrosis as the cause of craniomandibular pain and dysfunction: A unifying concept. J Oral Maxillofac Surg 47: 249-256.

19. Tanaka E, Detamore MS, Marcuri LG (2008): Degenerative disorders of the temporomandibular joint: etiology, diagnosis, and treatment. J Dent Res 87: 296-307.

20. de Leeuw R (2008): Internal derangements of the temporomandibular joint. Oral Maxillofac Surg Clin North Am 20: 159-168.

21. Fernandes JC, Martel-Pelletier J, Pelletier JP (2002): The role of cytokines in osteoarthritis pathophysiology. Biorheology 39: 237-246.

22. Kaneyama K, Segami N, Sun W, et al. (2005): Analysis of tumor necrosis factor-alpha, interleukin-6, interleukin-1beta, soluble tumor necrosis factor receptors I and II, interleukin-6 soluble receptor, interleukin-1 soluble receptor type II, interleukin-1 receptor antagonist, and protein in the synovial fluid of patients with temporomandibular joint disorders. Oral Surg Oral Med Oral Pathol Oral Radiol Endod 99: 276-284.

23. Kubota E, Kubota T, Matsumoto J (1998): Synovial fluid cytokines and proteinases as markers of temporomandibular joint disease. J Oral Maxillofac Surg 56: 192-198;

24. Kubota E, Imamura H, Kubota T, et al. (1997): Interleukin 1 beta and stromelysin (MMP3) activity of synovial fluid as possible markers of osteoarthritis in the temporomandibular joint. J Oral Maxillofac Surg 55: 20-27.
25. Kaneyama k, Segami N, Nishimura M, et al. (2002): Importance of proinflammatory cytokines in synoviall fluid from 121 joints with temporomandibular disorders. Br J Oral Maxillofac Surg 40: 418-423.

26. Fang PK, Ma XC, Ma DL, Fu KY (1999): Determination of interleukin-1 receptor antagonist, interleukin-10, and transforming growth factor-beta1 in synovial fluid aspirates of patients with temporomandibular disorders. J Oral Maxillofac Surg 57: 922-928.

27. Milam SB, Schmitz JP (1995): Molecular biology of temporomadibular joint disorders: proposed mechanisms of disease. J Oral Maxillofac Surg 53: 1448-1454.

28. Sato J, Segammi N, Kaneyama K, et al. (2004): Relationship of calcitonin gene-related peptide in synovial tissues and temporomandibular joint pain in humans. Oral Surg Oral Med Oral Pathol Oral Radiol Endod 98: 533-540.

29. Sato J, Segami N, Yoshitake Y, et al. (2007): Specific expression of substance $\mathrm{P}$ in synovial tissues of patients with asymptomatic non-reducing internal derangement of the temporomandibular joint: comparison with clinical findings. Br J Oral Maxillofac Surg 45: 372-377.

30. Adamski Z, Dudziak M, Zakrzewska K (2011): Etanercept in dermatological practice - authors' own experience in the treatment of psoriasis vulgaris and psoriatic arthritis. Post Dermatol Alergol 6: 435-441.

31. Kubota T, Kubota E, Matsumoto A, et al. (1998): Identification of matrix metalloproteinases (MMPs) in synovial fluid from patients with temporomandibular disorder. Eur J Oral Sci 106: 992-998.

32. Kacena MA, Marrel GA, Konda SR, et al. (2001): Inflammation and bony changes at the temporomandibular joint. Cells Tissues Organs 169: 257-264.

33. Donovan TE, Anderson M, Becker W, et al. (2011): Annual review of selected scientific literature: report of the committee in scientific investigation of the American Academy of Restorative Dentistry. J Prosthet Dent 106: 224-265.

34. Lee MC, Kawai Y, Shoji H, et al. (2004): Evidence of reactive oxygen species generation in synovial fluid from patients with temporomandibular disease by electron spin resonance spectroscopy. Redox Rep 9: 331-336. 